\title{
Research
}

\section{Contribution of paramedics in primary and urgent care:}

\author{
a systematic review
}

\begin{abstract}
\section{Background}

Within the UK, there are now opportunities for paramedics to work across a variety of healthcare settings away from their traditional ambulance service employer, with many opting to move into primary care.
\end{abstract}

\section{Aim}

To provide an overview of the types of clinical roles paramedics are undertaking in primary and urgent care settings within the UK.

\section{Design and setting \\ A systematic review.}

\section{Method}

Searches were conducted of MEDLINE, CINAHL, Embase, the National Institute for Health and Care Excellence, the Journal of Paramedic Practice, and the Cochrane Database from January 2004 to March 2019 for papers detailing the role, scope of practice, clinician and patient satisfaction, and costs of paramedics in primary and urgent care settings. Free-text keywords and subject headings focused on two key concepts: paramedic and general practice/primary care.

\section{Results}

In total, 6765 references were screened by title and/or abstract. After full-text review, 24 studies were included. Key findings focused on the description of the clinical role, the clinical work environment, the contribution of paramedics to the primary care workforce, the clinical activities they undertook, patient satisfaction, and education and training for paramedics moving from the ambulance service into primary care.

\section{Conclusion}

Current published research identifies that the role of the paramedic working in primary and urgent care is being advocated and implemented across the UK; however, there is insufficient detail regarding the clinical contribution of paramedics in these clinical settings. More research needs to be done to determine how, why, and in what context paramedics are now working in primary and urgent care, and what their overall contribution is to the primary care workforce.

\section{Keywords}

allied health personnel; ambulatory care extended roles; paramedics; primary care: urgent care.

\section{INTRODUCTION}

Within the UK, there are now opportunities for paramedics to work across a variety of healthcare settings, away from their traditional ambulance service employer These settings include acute hospital trusts, forensic health care, minor injury units, GP services, and urgent care centres. ${ }^{1-4}$

The NHS Long Term Plan ${ }^{5}$ further advocates the use of paramedics in primary care and the new 5-year framework for general practice contracts outlines funding support for 'first contact community paramedics' to be employed within primary care settings from 2021..$^{5}$ This may be appealing to some paramedics wishing to further develop their professional practice in this setting: ${ }^{6}$ therefore, they are among the group of allied health professionals who are attractive to GP surgeries based on this funding. As paramedics transition into these roles within primary care, their knowledge and skill set will undoubtedly change and grow, ${ }^{7-9}$ offering career development outside of the ambulance service.

The aim of this review was to describe the key reported findings from the current published UK literature available in this field, and to identify gaps in the evidence base since the last systematic review on the subject. ${ }^{10}$

\section{METHOD}

This is a systematic review, conducted using guidance from the Joanna Briggs

G Eaton, clinical doctoral fellow, MSc, PGCert, MCPara, FHEA; G Wong, clinical research fellow, MA, MD (Res), MRCGP, FHEA; KR Mahtani, GP and associate professor, PhD, PGDip, FRCGP, Nuffield Department of Primary Care Health Sciences,

University of Oxford, Oxford. V Williams, honorary researcher, PhD, RGN; N Roberts, Bodleian outreach librarian and information specialist. Bodleian Health Care Libraries, Knowledge Centre, Oxford.

\section{Address for correspondence}

Georgette Eaton, Nuffield Department of Primary Care Health Sciences, Radcliffe Primary Care
Institute. ${ }^{11}$ Findings are reported according to the extension for Preferred Reporting Items for Systematic Reviews and MetaAnalyses (PRISMA) guidelines. ${ }^{12}$ Prior to commencing this review, a study protocol was developed and registered with PROSPERO (ref: CRD42018109414).

\section{Information sources}

A sensitive search strategy was developed for MEDLINE (Ovid, January 2004 to third week of January 2019) and then translated to other databases. These were CINAHL (EBSCO, January 2004 to third week of January 2019); Embase (Ovid, January 2004 to third week of January 2019); National Institute for Health and Care Excellence (January 2004 to first week of February 2019); and Cochrane Database of Systematic Reviews (Cochrane Library, Wiley; January 2004 to third week of January 2019). Reference lists were screened and forward citation searches conducted for included studies. The Journal of Paramedic Practice (January 2004 to first week of March 2019) was hand-searched.

\section{Search strategy}

Databases were searched using free-text keywords and subject headings for the two key concepts: paramedic and general practice/primary care (see Supplementary Box S1 for details). Search terms describing scope of practice were not added because

Building, Radcliffe Observatory Quarter, University of Oxford, Oxford OX2 6GG, UK.

Email: Georgette.eatondstcatz.ox.ac.uk

ageorgette_eaton

Submitted: 7 October 2019; Editor's response:

3 November 2019; final acceptance:

5 December 2019.

(CThe Authors

This is the full-length article (published online 19 May 2020) of an abridged version published in print. Cite this version as: Br J Gen Pract 2020; DOI: https://doi.org/10.3399/bjgp20X709877 


\section{How this fits in}

Encouraged by the recent NHS Long Term Plan and the new 5-year framework for GP contracts, paramedics are being employed within primary care settings across the UK. The evidence within this review identifies that the role of the paramedic working in primary care is being advocated and implemented across the UK, with a greater range of autonomy and higher education levels. However, there is insufficient detail within the published literature regarding the clinical contribution paramedics make to the primary and urgent care workforce.

the aim was to retrieve all studies focusing on paramedics in primary care, regardless of clinical presentation or assessment. The search was limited from January 2004, to capture studies published since the last systematic review on the subject. ${ }^{10}$ No methodological filters or language limits were applied.

\section{Study selection}

A priori definitions and justifications for the screening criteria are outlined in Supplementary Box S2. Studies were selected for inclusion against the following criteria:

- Population: paramedics working within the UK only.

- Concept: information relating to role, scope of practice, work within health and social care systems, patient and carer satisfaction, clinician satisfaction, and costs.

- Context: working fully or partly in primary care settings, out-of-hours, urgent care centres, minor injury units, or walk-in centres.

- Study design: any literature lquantitative or qualitative) that presents information relevant to the purpose of this review was considered for inclusion.

Titles and abstracts were screened in order to include or exclude records according to eligibility criteria. Duplicate studies were excluded using reference management software Mendeley (version 1.19.4). The full text of potentially eligible studies was retrieved and originally assessed for eligibility by one author. Fulltext studies were independently screened by a second author. Comparisons of study selections were made between both authors. Where any discrepancy arose, they were discussed with a third author until a consensus had been reached.

\section{Data extraction}

To extract data from included studies, an amended data extraction form was adapted from the Joanna Briggs Institute's standardised template ${ }^{11}$ and included:

- objectives;

- questions;

- population;

- concept;

- context;

- study methodology;

- study citation; and

- findings relating to the themes of the previous review, ${ }^{10}$ including role, scope of practice, education, and commissioning.

Information was obtained from published studies. No authors were contacted for further information as reported methods and results were clear. Data extraction was undertaken by one author and discussed among the remaining authors.

\section{Analysis}

Data from all included studies were discussed between all authors. The included studies were described and summarised in a tabular form. Then, a coding framework was iteratively developed to present the key findings from the data extraction. A narrative synthesis was used to describe the nature and scope of the reported findings within the studies identified. In keeping with this methodology, quality appraisal of included studies was not carried out."

\section{RESULTS}

A total of 6765 citations were identified through database searching (see Supplementary Figure S1 for details). Following screening of titles and abstracts against the inclusion and exclusion criteria, 202 full-text studies were retrieved. After applying inclusion and exclusion criteria, 24 relevant full-text studies were identified. No new studies that had not already been considered were found upon screening the reference lists of these studies. The main reasons for exclusion were studies that reviewed practice outside of the UK $(n=137)$; where the paramedic was working within the ambulance service only ( $n=22)$; and where the population could not be identified as being a paramedic because of the term 'emergency care practitioner' encompassing both nurse and paramedic 
disciplines $(n=14)$. There was complete agreement on inclusion/exclusion decisions from independent screening against eligibility criteria at the full-text stage.

\section{Charting the results}

For the characteristics of the 24 included studies selected for review lall of which were published in English) see Supplementary Table S1. Study design varied across the included studies, with seven including primary research, ${ }^{13-19}$ two of secondary research, ${ }^{20,21}$ and the remaining comprising either a case study $(n=4), 22-25$ commentary $(n=4), 26-29$ report or strategic plan $(n=6), 5,30-$ ${ }^{34}$ or protocol for future research $(n=1)$. $^{35}$

Key findings were iteratively grouped into the following headings:

- description of the clinical role

- clinical work environment;

- reduction in GP workload;

- patient satisfaction;

- clinical activities in primary care; and

- education and training.

Description of the clinical role. Across the studies, the job title of the paramedic working in primary care differed, either being 'paramedic', 16,23,27-30,32,34 'specialist paramedic', 18,19,24 'paramedic practitioner', 14,17,22,36 'emergency care practitioner', 14,21,26,35 'advanced paramedic', 32,33 or 'community paramedic'. 5,31,33

The variety of titles was reported to be confusing for patients in two studies, ${ }^{13,17}$ as well as other clinicians within primary care, ${ }^{19,21,29}$ with concerns relating to the role, scope of practice, and autonomy of these paramedics.

Clinical work environment. In addition to variation in title, variation in clinical environment, in which paramedics were deployed,wasalsofound. Thestudiesreported paramedics working in commissioned home-visiting services, 14,17,18,25,30,35 minor units $15,20,21,24,30$ (including first aid units, minor injury units, minor departments in hospitals, and walk-in-centres), general practice, $5,14,15,19,22,27,28,31,33,34$ and out-ofhours. ${ }^{13,16,24}$

In some studies, paramedics retained the ambulance service as their main employer and rotated through primary care settings. ${ }^{15,18-21,24,29}$

Reduction in GP workload. All studies reviewed reported the paramedic contributing to the primary care workforce through working within a multidisciplinary team. In some cases, the role of paramedics in primary care settings was specifically reported to decrease GP workload by assessing and treating urgent, non-complex patients. $5,18,22,23,27,28$ However, two studies highlighted that patients may still prefer to see their registered GP, rather than a paramedic. ${ }^{17,18}$

Another two of the included studies raised the issue that patients may not have a straightforward consultation with a paramedic if treatment needed to be clarified with a medical doctor, 14,15 thereby adding an extra step to the patient journey. In some areas, there was an expectation that the paramedic report to a more senior clinician for all patient cases. ${ }^{23,26,33}$ Such referrals were reported as lengthening consultation time with little gain to the patient, and the paramedic role in these cases is of more limited value.

Other studies highlighted that the time paramedics spent with patients was generally longer than their physician or nursing counterparts within primary care, $13,15,16,19,21$ and so researchers questioned the benefit of their attendance from an economic standpoint. The slightly longer home-visits by paramedics was deemed a positive by patients in one study. ${ }^{17}$ Another study ${ }^{21}$ showed that the length of patient contact differed between clinical settings, typically being longer when paramedics were employed by ambulance services rather than in primary care. Reasons for this were not explored in the study, but it is worth noting that the pressure to see patients within a specific timeframe does not exist in the ambulance service, unlike in primary care.

Only one study associated paramedic roles in primary care with an overall cost saving, ${ }^{15}$ although this finding was an estimate and the study was authored in 2006; therefore, its relevance to today's NHS is ambiguous.

Patient satisfaction. Three studies used retrospective comparators ${ }^{37}$ to record patient satisfaction following treatment by a paramedic. 13,17,18 Although these studies document high satisfaction levels from patients who were visited by a paramedic in their home, in two studies a small minority of patients remained keen to be assessed by their GP, ${ }^{17.18}$ and other patients remained unclear about the purpose of the assessment undertaken by the paramedic. ${ }^{13}$

Clinical activities in primary care. The role of the paramedic in primary care varied little 
across studies, highlighting that paramedics who undertake roles in primary care lunder whichever title) generally undertake similar roles that focus on the undifferentiated, undiagnosed patient. These include minor injury and illness clinics, 15,19,22-24,28,33,34 home-visiting, 14,17-19,22,23,25,28,30,33,35 and using paramedic-specific skills (such as 12-lead electrocardiogram [ECG] interpretation) to assist in general health assessment. 22-24,28,29,31,34 There was no mention of paramedics providing highacuity care within these settings, but it was acknowledged that one of the advantages brought by paramedics was their ability to provide high-acuity or emergency care. $23,24,27,28$

Four case studies ${ }^{14,22-24}$ and one report ${ }^{34}$ presented typical days for paramedics working in primary care settings of GP surgeries and a rural first aid unit. The type of work undertaken across these case studies is similar, indicating that paramedics working in clinical primary and urgent care roles tend to adopt a similar working day.

Education and training. Education standards for paramedics working in primary and urgent care were explicitly mentioned in eight studies. 14-16,19,21,26,34,38 However, none of the publications by NHS England $d^{5,25,31}$ considered elements relating to education for paramedics working in primary and urgent care. Postgraduate education was outlined as a requirement for paramedics to work in primary and urgent care, ${ }^{14-16,19,21,26,34,38}$ in line with the guidance outlined by the College of Paramedics; ${ }^{39}$ however, there was little detail on the specific competencies required to work in these settings. One study ${ }^{16}$ highlighted the importance of supervision and mentoring for paramedics as they entered primary or urgent care roles, whereas another ${ }^{22}$ outlined how paramedics may also be used to provide mentorship to training physician assistants and nurses (for a brief summation of these findings, see Supplementary Box S3).

\section{DISCUSSION}

\section{Summary}

This review has set out the scope and content from the literature, published since the seminal study on the subject in $2005 .{ }^{10}$ In the time since that review was published, the role of the ambulance service has changed substantially, and the role of the paramedic has subsequently evolved. Now, just $8 \%$ of 999 emergency ambulance calls are for people with life-threatening illnesses or injuries, ${ }^{38}$ suggesting that a large proportion of patients access the ambulance service with lower-acuity presentations. This has subsequently caused a change in what is expected of a paramedic. As well as emergency care and advanced life support, paramedics now need to be skilled in managing acute-on-chronic long-term conditions, acute presentations of mental ill-health, social care assessments, and a range of undifferentiated urgent care presentations. ${ }^{7.8}$ New legislation supports non-medical prescribing by advanced paramedics, allowing these paramedics to 'complete' patient care and avoid referrals for secondary assessments and treatment. With changing demand on the health and social care services, more higher education thresholds, and social dependence on the ambulance service, ${ }^{40}$ the modern paramedic has evolved to become a generalist.

\section{Strengths and limitations}

These results should be considered in the light of some limitations. Despite creating and implementing a search strategy with the support of an information specialist, it is possible that relevant studies were missed. A decision was made to describe and map the evidence base. It was decided not to conduct formal meta-analysis exploring the effectiveness of paramedics in primary and urgent care, nor to critically assess the quality of the research.

\section{Comparison with existing literature}

There is some evidence in this review to suggest that paramedics may be able to apply their extended skills to safely assess and treat patients in primary care ${ }^{20}$ and that the role of paramedics working in primary and urgent care settings is received positively. ${ }^{13,17,19,20,28}$ However, there is also evidence which demonstrates that patients perceive the role and responsibilities of paramedics in primary care settings as unclear, ${ }^{13,17}$ perhaps not helped by the differences in title and scope of practice across clinical settings. ${ }^{14,21}$ The College of Paramedics has made distinctions between 'paramedics', 'specialist paramedics', and 'advanced paramedics', ${ }^{39}$ and advocated against the use of the term emergency care practitioner' to describe paramedics in extended roles. This review highlights both the variety of job titles paramedics working in primary and urgent care operate under, as well as the lack of uptake of the professional body's recommendations on the job titles of registered paramedics. It is interesting to note that the two most recent documents by NHS England ${ }^{5,31}$ refer to 
paramedics working within primary care under two different job titles l'first contact community paramedic' and 'community paramedic'), neither of which are endorsed nor used by the professional body.

This review also indicates the variety of settings in which paramedics are working, and the broadly similar roles paramedics are employed in. Within these diverse settings paramedics are likely to encounter patients presenting with conditions of lower acuity. Such patients may not fully utilise the unique skill sets of seeing paramedics, who on the whole are trained to manage urgent and emergency presentations. . $4,27,28,34^{2}$

\section{Implications for research and practice}

What is clear from this review is the lack of standardisation across the UK. For example, the role of the paramedic in Northumberland ${ }^{33}$ and Salford ${ }^{22}$ is broadly similar to the role in Oxfordshire ${ }^{24}$ in terms of scope of practice, but there are differences in the level of autonomy. The in-hours Northumberland model relies on GPs retaining oversight and control, whereas the in-hours model in Salford promotes greater autonomy, as does the out-of-hours role in Oxfordshire. There is no differentiation of autonomy in studies where paramedics worked in minor injury units, 15,20,21,30 and paramedics who rotated from the ambulance service were still expected to report to the senior clinician (usually the GP) while in the primary care setting. ${ }^{16,19,32,34}$ This was generally attributed to the inability of paramedics to prescribe medicines at the time these studies were written. ${ }^{22,28}$ The level of autonomy afforded to paramedics working in these settings requires further investigation if paramedics are to be employed optimally in the NHS. Although postgraduate education is deemed a necessity to work in primary and urgent care settings ${ }^{14-16,19,21,26,34,38,39}$ the lack of standardisation makes the development of training programmes to prepare paramedics to work in these settings difficult.

The first 5-year framework for GP

\section{contracts initially outlined paramedics}

working in primary care should be paid at Agenda-for-Change Band $6,{ }^{31}$ yet the study by Turner and Williams ${ }^{19}$ found that Band 6 posts were considered training posts with supervision and mentoring within primary and urgent care. The most recent update has outlined that paramedics, included into the reimbursement scheme from April 2021, will be reimbursed up to an indicative Agenda-for-Change Band 7 rate. ${ }^{41}$ The update further outlined that, to qualify as an advanced paramedic practitioner, a relevant Master's degree is the level of education required for this role. This more accurately represents the degree of autonomy paramedics can have, and also outlines the range of patients they can safely manage. However, this document continues to conflate titles, using both terms 'community paramedic' and 'advanced paramedic practitioners'.

Although several studies make the claim that paramedics in primary care may provide cost savings, only one study provided any empirical data to back up this claim and the data were only estimates. ${ }^{15}$ Further evidence needs to be gathered to determine whether paramedics in primary care are able to reduce costs, perhaps by directing GP time to more complex patients with chronic conditions, and to managing both lower- and higher-acuity same-day presentations autonomously.

The evidence within this review identifies that the role of the paramedic working in primary and urgent care is being advocated and implemented across the UK, but fails to provide sufficient detail regarding the clinical contribution of paramedics in this clinical setting. If primary care employers are to see the full potential of paramedics working in these settings, more research needs to be done to determine how, why, and in what context paramedics are now working in primary and urgent care, and what their overall contribution is to the primary care workforce.
Georgette Eaton would like to thank Rachel Gardner, DPhil Student at the University of Oxford, for second-screening extracted articles.

\section{Open access}

This article is Open Access: CC BY-NC 4.0 licence (http://creativecommons.org/ licences/by-nc/4.0/).

\section{Discuss this article}

Contribute and read comments about this article: bjgp.org/letters 


\section{REFERENCES}

1. Evans R, McGovern R, Birch J, Newbury-Birch D. Which extended paramedic skills are making an impact in emergency care and can be related to the UK paramedic system? A systematic review of the literature. Emerg Med J 2014; 31(7): 594-603.

2. Mahtani KR, Eaton G, Catterall M, et al. Setting the scene for paramedics in general practice: what can we expect?. J R Soc Med 2018; 111(6): 195-198.

3. O'Meara P. Community paramedics: a scoping review of their emergence and potential impact. Int Paramed Pract 2014; 4(1): 5-12.

4. Williams B, Fielder C, Strong G, et al. Are paramedic students ready to be professional? An international comparison study. Int Emerg Nurs 2015; 23(2): $120-126$

5. NHS England. The NHS Long Term Plan. 2019. wuw.longtermplan.nhs.uk laccessed 6 Apr 2020).

6. Eaton G, Mahtani K, Catterall M. The evolving role of paramedics - a NICE problem to have?. J Health Serv Res Policy 2018; 23(3): 193-195.

7. National Institute for Health and Care Excellence. Paramedics with enhanced competencies. In: Emergency and acute medical care in over 16s: service delivery and organisation. London: NICE, 2017. https://www.nice.org.uk/ guidance/gid-cgwave0734/documents/draft-guideline-3 (accessed 6 Apr 2020).

8. National Institute for Health and Care Excellence. Paramedic remote support. In: Emergency and acute medical care in over 16s: service delivery and organisation. London: NICE, 2017. https://www.nice.org.uk/guidance/GIDCGWAVE0734/documents/draft-guideline-4 (accessed 6 Apr 2020).

9. Primary Care Workforce Commission. The future of primary care: creating teams for tomorrow. 2015. https://www.hee.nhs.uk/sites/default/files/ documents/The\%20Future\%20of\%20Primary\%20Care\%20report.pdf laccessed 6 Apr 2020)

10. Ball L. Setting the scene for the paramedic in primary care: a review of the literature. Emerg Med J 2005; 22(12): 896-900.

11. Peters M, Godfrey C, Mclnerney P, et al. Scoping reviews. In: Aromataris E, Munn Z, eds. Joanna Briggs Institute reviewer's manual. 2017. https:// reviewersmanual.joannabriggs.org (accessed 6 Apr 2020).

12. Tricco AC, Lillie E, Zarin W, et al. PRISMA extension for Scoping Reviews (PRISMA-ScR): checklist and explanation. Ann Intern Med 2018; 169(7): 467473.

13. Halter M, Marlow T, Mohammed D, Ellison GTH. A patient survey of out-ofhours care provided by emergency care practitioners. BMC Emerg Med 2007; 7: 4 .

14. Lattimer V, Burgess A, Knapp F, et al. The impact of changing workforce patterns in emergency and urgent out-of-hours care on patient experience, staff practice and health system performance: final report for the National Institute for Health Research Service Delivery and Organisation programme. 2010. http://www.netscc.ac.uk/hsdr/files/project/SDO_FR_08-1519-97_V01.pdf laccessed 6 Apr 2020).

15. Mason S, Coleman P, O'Keeffe C, et al. The evolution of the emergency care practitioner role in England: experiences and impact. Emerg Med J 2006; 23(6): 435-439.

16. Moule P, Clompus S, Lockyer L, et al. Preparing non-medical clinicians to deliver GP out-of-hours services: lessons learned from an innovative approach. Educ Prim Care 2018; 29(6): 376-380.

17. Proctor A. Home visits from paramedic practitioners in general practice: patient perceptions. J Paramed Pract 2019; 11(3): 115-121.

18. RSM UK Group. Better local care Hampshire multispecialty community provider vanguard. Deep dive evaluation report: paramedic home visiting service. RSM UK Group, 2017

19. Turner J, Williams J. An evaluation of early stage development of rotating paramedic model pilot sites. 2018. https://uhra.herts.ac.uk/bitstream/ handle/2299/21174/Rotating_Paramedics_Final_Report.pdf? laccessed 6 Apr 2020).

20. Turner J, Coster J, Chambers D, et al. What evidence is there on the effectiveness of different models of delivering urgent care? A rapid review. Health Serv Deliv Res 2015; 3(43): 1-160.
21. Hill H, McMeekin P. Price C. A systematic review of the activity and impact of emergency care practitioners in the NHS. Emerg Med J 2014; 31(10): 853-860.

22. Brown P. A day in the life of a paramedic advanced clinical practitioner in primary care. J Paramed Pract 2017; 9(9): 378-386.

23. Daly J. The paramedic in the community: my story. Prim Health Care 2013; 22(9): 16-19.

24. Eaton G. Taking healthcare to the community: the evolving role of paramedics. J Paramed Pract 2017; 9(5): 190-191.

25. NHS England. Practice based paramedics, S Kent coast. 2017. https://umw. england.nhs.uk/wp-content/uploads/2016/03/releas-capcty-case-study-4-138. pdf (accessed 6 Apr 2020).

26. Scott J, Carney C. Emergency care practitioners. Emerg Med J 2004; 21(3): 273-274.

27. Spence D. Bad medicine: good medicine - the GP paramedic. Br J Gen Pract 2017; DOI: https://doi.org/10.3399/bjgp17X691445.

28. Spencer M. Skill mix: how paramedics can help general practice. GPonline 2016; 25 May: https://www.gponline.com/skill-mix-paramedics-help-generalpractice/article/1396392 (accessed 6 Apr 2020).

29. Woollard M. The role of the paramedic practitioner in the UK. J Emerg Prim Health Care 2006; 4(1)

30. NHS England. Prime Minister's challenge fund: improving access to general practice. First evaluation report: October 2015. 2015. https://www.england.nhs. uk/wp-content/uploads/2015/10/pmcf-wv-one-eval-report.pdf laccessed 6 Apr 2020).

31. NHS England. Investment and evolution: a five-year framework for GP contract reform to implement The NHS Long Term Plan. 2019. https://www.england. nhs.uk/wp-content/uploads/2019/01/gp-contract-2019.pdf laccessed 6 Apr 2020).

32. NHS Wales. Our plan for a primary care service for Wales up to March 2018 2014. http://www.wales.nhs.uk/sitesplus/documents/986/our\%20plan\%20 for\%20primary\%20care\%20in\%20wales\%20up\%20to\%20march\%202018.pdf (accessed 6 Apr 2020)

33. Northumberland Local Medical Committee, Northumberland Clinical Commissioning Group. A primary care strategy for Northumberland 2016-2020: a vision for a vibrant and sustainable future for primary care. 2016.

34. NHS Fareham and Gosport and South Eastern Hampshire Clinical Commissioning Groups. A guide for general practice employing a paramedic. 2018. http://www.wessexdeanery.nhs.uk/pdf/2018\%2006\%2005\%20 Employing\%20a\%20Paramedic\%20in\%20Primary\%20Care\%20Toolkit\%20v4. pdf (accessed 6 Apr 2020).

35. Abrams R, Wong G, Mahtani KR, et al. Understanding the impact of delegated home visiting services accessed via general practice by community-dwelling patients: a realist review protocol. BMJ Open 2018; $8(11)$ : e024876.

36. NHS England. Allied health professions into action: using allied health professionals to transform health, care and wellbeing 2016/17-2020/21. 2017. https://www.england.nhs.uk/wp-content/uploads/2017/01/ahp-actiontransform-hlth.pdf laccessed 6 Apr 2020).

37. Pinnock H, Epiphaniou E, Pearce G, et al. Implementing supported selfmanagement for asthma: a systematic review and suggested hierarchy of evidence of implementation studies. BMC Med 2015; 13: 127.

38. Keogh B. Ambulance response programme. 2017. https://wnw.england.nhs. uk/wp-content/uploads/2017/07/ambulance-response-programme-letter.pdf (accessed 6 Apr 2020).

39. College of Paramedics. Digital career framework. 2017. https://wnw. collegeofparamedics.co.uk/COP/Professional_development/Digital_Career_ Framework/COP/ProfessionalDevelopment/Digital_Career_Framework. aspx?hkey=5058228a-13ef-4d38-a7b0-255e6263c9f7 (accessed 15 Apr 2020).

40. Wankhade P. Cultural characteristics in the ambulance service and its relationship with organisational performance: evidence from the UK. 2010. https://www.academia.edu/920660/Cultural_characteristics_in_the ambulance_service_and_its_relationship_with_organisational_performance evidence_from_the_UK (accessed 6 Apr 2020).

41. NHS England. Update to the GP contract agreement 2020/21-2023/24. 2020. https://www.england.nhs.uk/wp-content/uploads/2020/03/update-to-the-gpcontract-agreement-v2-updated.pdf (accessed 15 Apr 2020). 